\title{
The Motor Activity of Mammalian Axonemal Dynein Studied In Situ on Doublet Microtubules
}

\author{
David P. Lorch, ${ }^{1 *}$ Charles B. Lindemann, ${ }^{2}$ and Alan J. Hunt ${ }^{1}$ \\ ${ }^{1}$ Department of Biomedical Engineering, University of Michigan, \\ Ann Arbor, Michigan \\ ${ }^{2}$ Department of Biological Sciences, Oakland University, \\ Rochester, Michigan
}

\begin{abstract}
Flagellar dynein generates forces that produce relative shearing between doublet microtubules in the axoneme; this drives propagated bending of flagella and cilia. To better understand dynein's role in coordinated flagellar and ciliary motion, we have developed an in situ assay in which polymerized single microtubules glide along doublet microtubules extruded from disintegrated bovine sperm flagella at a $\mathrm{pH}$ of 7.8. The exposed, active dynein remain attached to their respective doublet microtubules, allowing gliding of individual microtubules to be observed in an environment that allows direct control of chemical conditions. In the presence of ATP, translocation of microtubules by dynein exhibits Michaelis-Menten type kinetics, with $V_{\max }=4.7 \pm 0.2 \mu \mathrm{m} / \mathrm{s}$ and $K_{\mathrm{m}}=124 \pm 11 \mu \mathrm{M}$. The character of microtubule translocation is variable, including smooth gliding, stuttered motility, oscillations, buckling, complete dissociation from the doublet microtubule, and occasionally movements reversed from the physiologic direction. The gliding velocity is independent of the number of dynein motors present along the doublet microtubule, and shows no indication of increased activity due to ADP regulation. These results reveal fundamental properties underlying cooperative dynein activity in flagella, differences between mammalian and non-mammalian flagellar dynein, and establish the use of natural tracks of dynein arranged in situ on the doublet microtubules of bovine sperm as a system to explore the mechanics of the dynein-microtubule interactions in mammalian flagella. Cell Motil. Cytoskeleton 65: 487-494, 2008. (c) 2008 Wiley-Liss, Inc.
\end{abstract}

Key words: flagella; kinetics; gliding assay; doublet microtubule; ADP

\section{INTRODUCTION}

Axonemal dynein is an extremely large multi-subunit motor protein. Current evidence suggests that each dynein $380 \mathrm{kDa}$ heavy chain contains a fully functional motor domain (the globular "head") that is composed of six AAA modules formed from 2350 residues of the Cterminal end of the chain [Mocz and Gibbons, 2001]. In flagella of the metazoans, which include mammalian sperm flagella, each outer dynein arm contains two heavy chains, and the arms repeat at $24 \mathrm{~nm}$ intervals along the doublet microtubules (dMTs). Six unique inner arms are arranged in a pattern that repeats every $96 \mathrm{~nm}$
Contract grant sponsor: National Science Foundation; Contract grant numbers: MCB-0334835 and MCB-0516181; Contract grant sponsor: NIH; Contract grant number: R01GM076177.

*Correspondence to: David P. Lorch; Lurie Biomedical Engineering Building, 1101 Beal Ave., Ann Arbor, MI 48109, USA. E-mail: dlorch@umich.edu

Received 17 October 2007; Accepted 9 March 2008

Published online 17 April 2008 in Wiley InterScience (www. interscience.wiley.com).

DOI: $10.1002 / \mathrm{cm} .20277$ 
[Goodenough and Heuser, 1985]. Five of these contain a single heavy chain and one inner arm has two (dynein I1 also called dynein $\mathrm{f}$ ). The dynein motors act as the primary force producers for flagellar and ciliary motion. Although it is well established that flagellar bending is induced by dynein shearing adjacent dMTs in the axoneme [Brokaw, 1989], the specifics of the dynein activation cycle that produces propagated oscillations of flagella remain to be determined. Although dynein was first recognized over 40 years ago [Gibbons and Rowe, 1965], there is much still to understand about its basic biochemistry and mechanics. The biochemistry of the dynein ATP-hydrolysis cycle has been studied in reduced assays [Oiwa and Sakakibara, 2005], and some studies of motility have been performed using dynein isolated from nonmammalian axonemes [Sakakibara et al., 1999; Hirakawa et al., 2000], but relatively little has been done to study mammalian, axonemal dynein in a reduced, chemically controlled environment. This is mostly because isolating functional dynein from mammalian axonemes has proven difficult. The structure of the mammalian axoneme has evolved several differences from non-mammalian axonemes such as the addition of a fibrous sheath, a mitochondrial sheath, and outer dense fibers. As mammalian dynein has been subject to relatively little study in reduced systems, it is unclear if further differences are present at the level of motor functioning.

To better understand axonemal dynein's role in the intricately coordinated motion of flagella and cilia, we use an in situ assay in which fluorescently labeled single microtubules (MTs) are observed gliding along dMTs from disintegrated bovine sperm flagella. This allows study of exposed, active dynein while they are still attached to their natural substrate: the dMTs, and presumably attached in a physiologic geometry as suggested by electron micrographs and tomography of the dMTs from Tetrahymena, Chlamydomonas, and sea urchin sperm [Sugrue et al., 1991; Avolio et al., 1986; Nicastro et al., 2006; Sui and Downing, 2006]. Variable MT translocation behaviors are observed including smooth gliding, stuttered motility, oscillations, buckling, occasionally complete dissociation from the dMT, and infrequent travel in the non-physiologic direction. The ATPdependent gliding velocity displays Michaelis-Menten kinetics, is independent of the number of dynein motors acting on a MT, and decreases at ADP concentrations of 100 to $500 \mu \mathrm{M}$.

\section{MATERIALS AND METHODS}

\section{Treatment of Bovine Sperm}

Preparation and use of bovine sperm for direct study of the mammalian axoneme follows established procedures. Removal of the membrane is performed by exposing the sperm to $0.1 \%$ Triton $\mathrm{X}-100$ detergent [Lindemann and Schmitz, 2001], and mitochondrial sheaths are removed using a freeze/thaw cycle [Lindemann et al., 1980]. When the structural integrity of bovine sperm is disrupted by removing their membranes and mitochondrial sheaths, the action of dynein motors in the presence of ATP no longer causes bending, but instead causes disintegration, and dMTs are extruded out of the proximal $12 \mu \mathrm{m}$ of the flagellum, where the mitochondrial sheath has been removed. This disintegration exposes dynein, which is still bound to the extruded dMTs.

\section{Preparation of Fluorescent MTs}

$\alpha-\beta$-Tubulin dimers are extracted from bovine brain by three cycles of polymerization and depolymerization and purification on a phosphocellulose column, then frozen [Weingarten et al., 1974; Howard et al., 1993]. Cycling is repeated to remove any denatured protein off the column. In some studies, tubulin is purified without a column using high-molarity cycling [Castoldi and Popova, 2003]. Tubulin is fluorescently labeled through addition of tetramethylrhodamine-succinimidyl ester, and again run through cycles of polymerization and depolymerization [Hyman et al., 1991]. GTP and $\mathrm{MgCl}_{2}$ is added to a mixture of labeled and unlabeled tubulin in BRB80 buffer and incubated at $37^{\circ} \mathrm{C}$ to polymerize the tubulin into MTs; subsequently taxol is added to stabilize the MTs for use.

\section{Gliding Assay}

Bovine sperm, with membranes and mitochondrial sheaths removed, are either left suspended in the demembranation solution and observed under a glass coverslip preparation (where the coverslip is simply dropped on top of the sample on the glass slide), or the solution is exchanged after the axonemes settle onto the cover slip of a chamber constructed of a cover slip and glass slide separated by two strips of double-sided tape. For the coverslip preparation observations, the demembranation solution $(20 \mathrm{mM}$ Tris buffer at a $\mathrm{pH}$ of $7.8,132 \mathrm{mM}$ sucrose, $24 \mathrm{mM}$ K-glutamate, $1 \mathrm{mM} \mathrm{MgSO}$, $1 \mathrm{mM}$ DTT, $0.1 \%$ Triton $\mathrm{X}-100,0.5 \mathrm{mM}$ EGTA, $5 \mathrm{mM}$ sodium citrate, and $10 \mu \mathrm{M}$ cAMP) is converted to motility buffer to observe MT gliding by adding $10 \mu \mathrm{M}$ taxol, anti-fade solution $(600 \mu \mathrm{g} / \mathrm{mL}$ glucose oxidase, $30 \mathrm{mM}$ glucose, and $120 \mu \mathrm{g} / \mathrm{mL}$ catalase) to prevent photodamage and bleaching, $1 \mathrm{mM} \mathrm{MgCl} 2$, ATP, and fluorescent MTs. The addition of ATP initiates disintegration of the axonemes and subsequently supports MT gliding along dMTs. For the chamber observations, the axonemes are disintegrated upon exchanging the demembranation solution with a disintegration solution of $50 \mathrm{mM}$ Tris buffer 
a
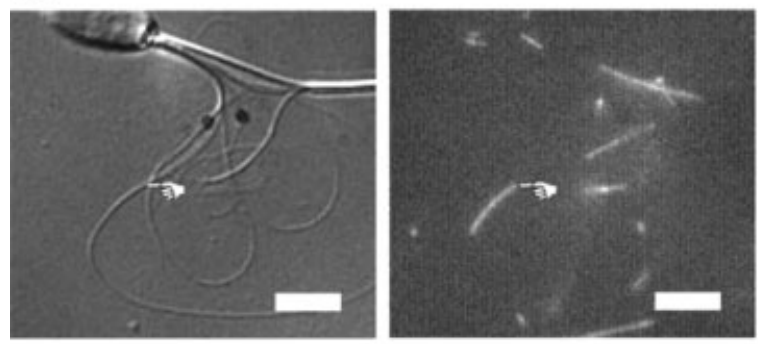

0 seconds

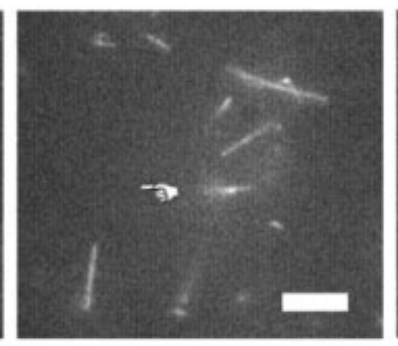

6.6 seconds

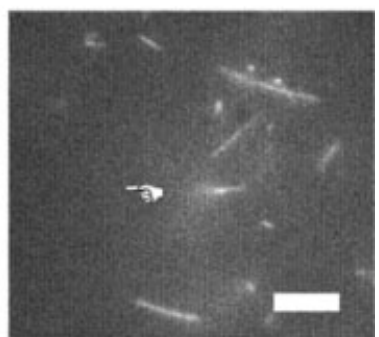

13.1 seconds

b

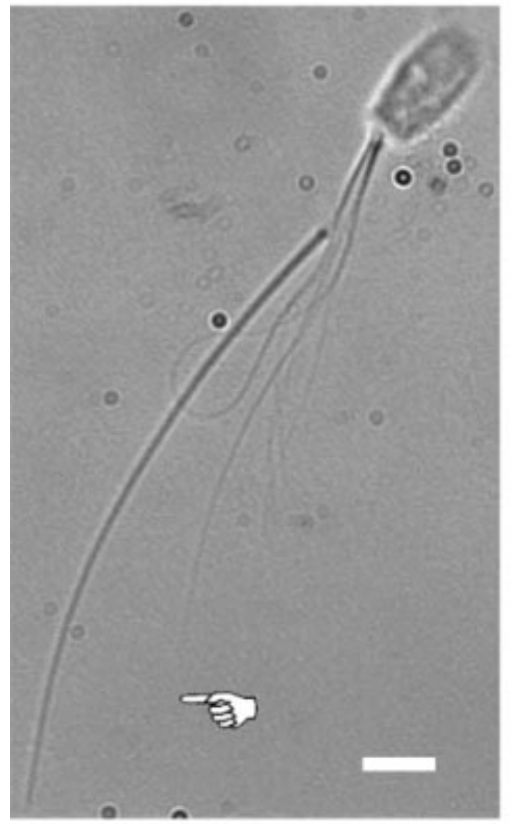

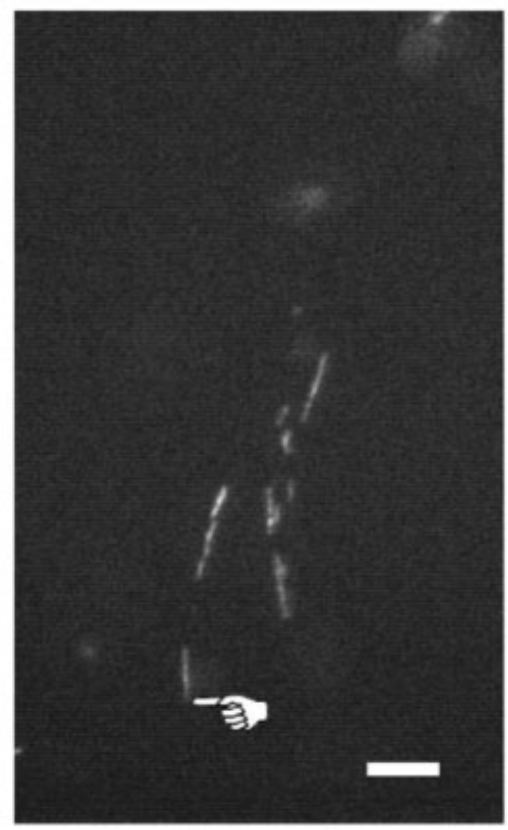

0 seconds

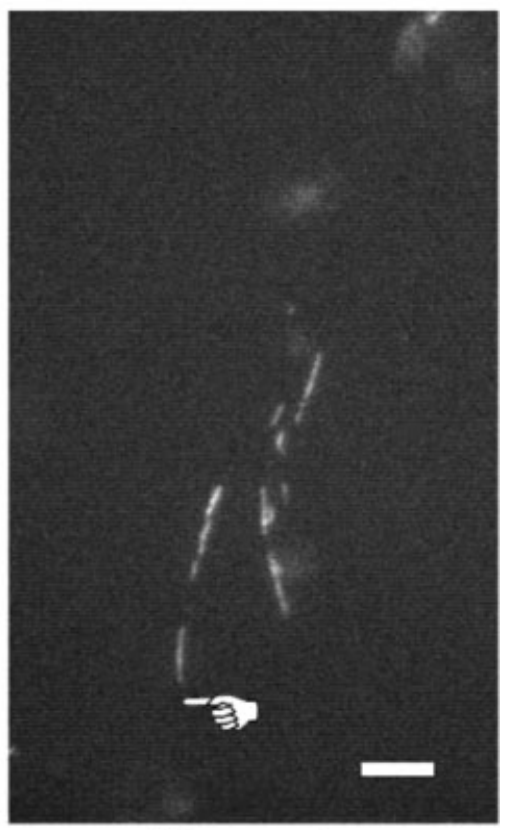

12 seconds
Fig. 1. Movement of fluorescent MTs along mammalian dMTs. These assays were performed at ATP concentrations of $0.05 \mathrm{mM}$ (a) and $1 \mathrm{mM}$ (b). The left panel in each sequence is a DIC image of the disintegrated axoneme. The following images are samples of fluorescent images (taken every 200 or $300 \mathrm{~ms}$ ) showing MT movement over time. The hand in each image points to the starting location of the moving MT. The MT in (a) is moving away from the base of the axoneme and did not move continuously, exhibiting starts and stops throughout its translocation. Prior to this MT's first stop, its velocity was $\sim 1.2 \mu \mathrm{m} / \mathrm{s}$. The MT in (b) is moving $\sim 1.4 \mu \mathrm{m}$ towards the base of the axoneme at a velocity of $\sim 0.1 \mu \mathrm{m} / \mathrm{s}$. Bar $=5 \mu \mathrm{m}$.

basal end of the flagella. For any data analysis involving MT length, the entire length of the MT was in contact with the dMT throughout the motion. this disintegration solution is exchanged with a motility buffer solution of $50 \mathrm{mM}$ Tris buffer ( $\mathrm{pH} 7.8$ ), $1 \mathrm{mM}$ EGTA, $10 \mu \mathrm{M}$ taxol, anti-fade solution, $1 \mathrm{mM} \mathrm{MgCl}_{2}$, ATP, $1 \mathrm{mM}$ DTT, and fluorescent MTs. The ATP in the motility buffer solutions is varied as detailed below. After adding motility buffer, samples are viewed under a microscope with DIC and fluorescent imaging capabilities. Images of fluorescent MT movement along dMTs are analyzed using ImageJ (an NIH open-source image processing and analysis software) to measure their gliding velocities. MT gliding velocities are calculated only for periods of constant motion moving away from the

\section{RESULTS}

We find that fluorescent MTs added to extruded dMTs immobilized on coverslips exhibit ATP-dependent gliding as they are propelled along rows of dynein motors on the dMTs. Movements were characterized by tracking the gliding MTs through a series of successive images (Fig. 1). The character of MT interactions with the dMTs is variable (Table I), but most commonly MTs associate laterally with dMTs all along the MTs' length, 
TABLE I. Frequency of MT Gliding Characteristics

\begin{tabular}{lc}
\hline MT gliding characteristic & $\%$ of gliding MTs $(N=879)$ \\
\hline Translocation towards the flagellar base & $<1 \%(7)$ \\
Oscillating & $\sim 2 \%(16)$ \\
Buckling & $<1 \%(8)$ \\
Stuttered motility & $\sim 9 \%(81)$ \\
\hline
\end{tabular}

and remain immobilized at the attachment location. Some MTs become attached at a point crossing the dMT and rotate back and forth around that point; often these MTs dissociate from the dMT after a period of attachment, and in some cases are translocated over the point of attachment. This rotation suggests these MTs may be attached to a single motor, and swivel about the motor as they are propelled. The MTs that glide along a dMT exhibit variable characteristics of motion. Often a gliding MT displays periods of motion interrupted with stops, producing a stuttering movement, or the MT stalls at the leading end, causing it to buckle as the trailing end continues to move. MTs would often glide past another immobilized MT on the dMT. This likely reflects the gliding MT being propelled along one set of either the inner or outer arm rows of dynein on the dMT, while the stationary MT was on the other row.

Notably, MT gliding sometimes proceeds in the non-physiologic direction toward the base of the axoneme (where the minus ends of the dMTs are located) (Fig. 1b), and occasionally the direction of MT gliding oscillates over short distances, switching between movement toward and away from the base of the axoneme. These behaviors are surprising, as in the normal state of a functional axoneme, dynein on one dMT pushes its adjacent dMT away from the basal end of the axoneme due to minus end directed activity of dynein motors. These variabilities may reflect properties of flagellar dynein that allow oscillatory bending of flagella, which require dMTs to cycle between sliding past each other in opposite directions.

Initially, gliding assays were performed in coverslip preparations in the same solution in which the bovine sperm were demembranated and stored. In this case MT gliding velocity exhibits a time-dependent decrease, slowing to half the initial speed within $5 \mathrm{~min}$. The decrease in gliding velocity can be prevented by adding an ATP regenerating system $(2 \mathrm{mM}$ phosphoenolpyruvate and $0.1 \mathrm{mg} / \mathrm{mL}$ pyruvate kinase) to the experimental solutions, indicating that slowing is due to depletion of ATP. This depletion is apparently due to MT-dependent ATPase activity, since when the axonemes and ATP are pre-incubated for $30 \mathrm{~min}$ after disintegration before MTs are added, the velocities are similar to gliding velocities immediately after disintegration. This unexpectedly large MT-dependent ATPase activity is much higher

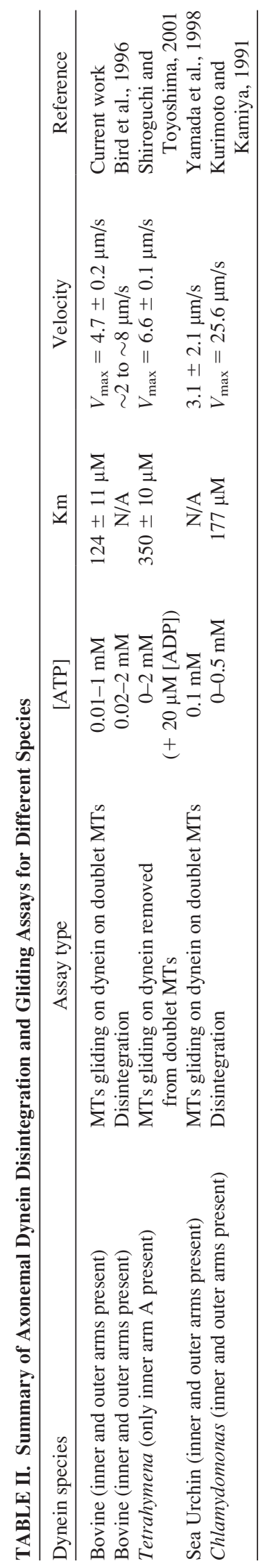




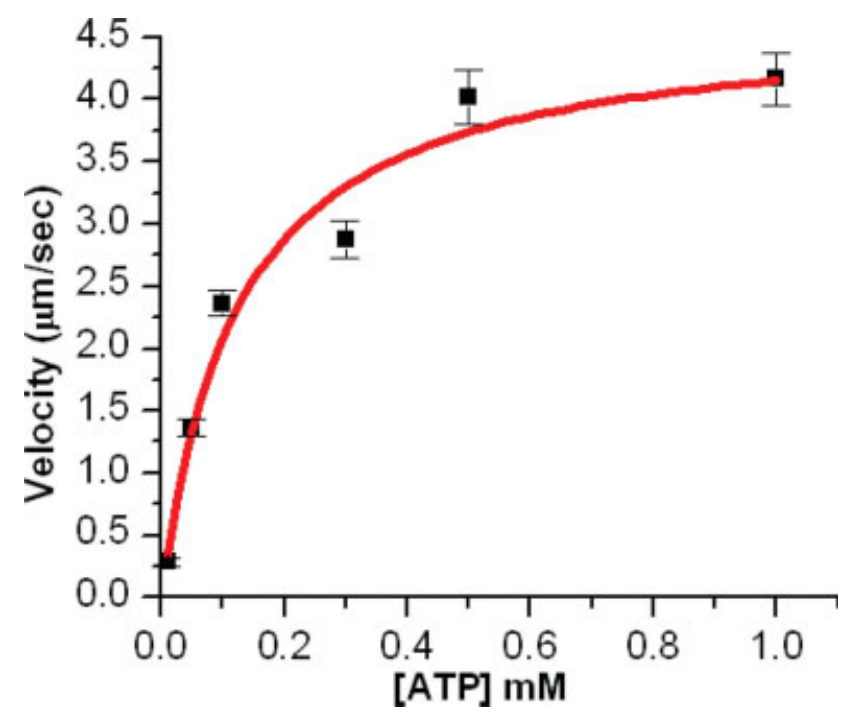

Fig. 2. MT gliding velocity $\pm \mathrm{SE}$ versus $[\mathrm{ATP}]$. Curve is fit to Michaelis-Menten, $V_{\max }=4.7 \pm 0.2 \mu \mathrm{m} / \mathrm{s}$ and $K_{\mathrm{m}}=124 \pm 11 \mu \mathrm{M}$. Each data point was calculated from 7 to 19 MT translocations.

than is expected from dynein activity alone; the total number of axonemal dyneins in an assay slide are of the order of a few billion, and even if all were actively hydrolyzing ATP at $100 \mathrm{~s}^{-1}$, the depletion of the $1 \mathrm{mM}$ ATP in the $7-\mu \mathrm{L}$ sample volume would be negligible. Thus to avoid ATP depletion by unidentified, presumably soluble MT-dependent ATPases, we switched to using flow chambers to allow fluid exchange to remove any soluble enzymes leftover from when the axonemes were demembranated. With this procedure, the MT gliding velocity does not decrease over time, indicating that rapid ATP depletion is indeed due to soluble enzymes, and allowing well-controlled study of the relation between gliding velocity and the ATP concentration.

ATP-dependent MT gliding follows MichaelisMenten kinetics, with a maximum velocity of $4.7 \pm 0.2$ $\mu \mathrm{m} / \mathrm{s}$ and a $K_{\mathrm{m}}$ of $124 \pm 11 \mu \mathrm{M}$ (Fig. 2). This is fairly consistent with axonemal dynein behavior observed in many mammalian [Bird et al., 1996] and non-mammalian [Kurimoto and Kamiya, 1991; Yamada et al., 1998; Shiroguchi and Toyoshima, 2001] systems as summarized in Table II. Since it has previously been proposed that ADP may regulate dynein's activity at a second allosteric binding site [Kinoshita et al., 1995; Shiroguchi and Toyoshima, 2001], we examined the velocity of MT gliding in the presence of ADP, finding decreased gliding velocity with the addition of 100 or $500 \mu \mathrm{M}$ ADP; velocities, respectively $1191 \pm 24 \mathrm{~nm} / \mathrm{s}$ and $1061 \pm 56$

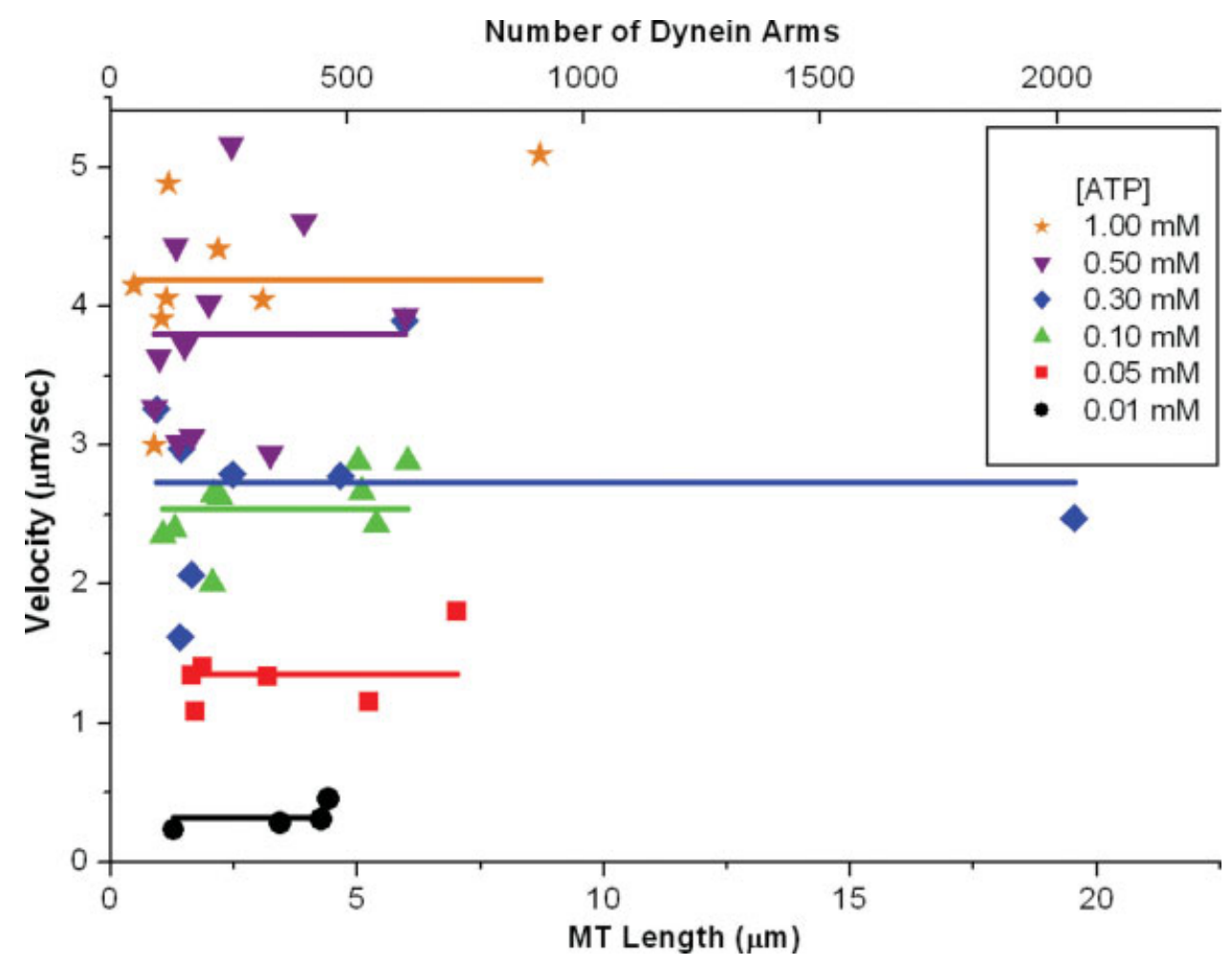

Fig. 3. MT gliding velocity is independent of length. The number of motors potentially propelling a MT was estimated by assuming 41.7 outer dynein arms ( 83 dynein heads) plus 62.5 inner dynein arms (73 dynein heads) along $1 \mu \mathrm{m}$ of dMT. Thus, the number of motors acting on a MT gliding along a dMT is expected to vary in proportion to the MT length. Lines are average velocity; the slopes of regression fits for each ATP concentration were not significantly different from zero. Each data point was calculated from 4 to 11 MT translocations. 
$\mathrm{nm} / \mathrm{s}$, are compared with the $1364 \pm 70 \mathrm{~nm} / \mathrm{s}$ at the same ATP concentration $(0.05 \mathrm{mM})$ without added ADP.

An advantage to examining motor activity on dMTs is that the motors are distributed with uniform spacing [Nicastro et al., 2006; Sui and Downing, 2006], and thus the number of motors acting on a MT is expected to scale in direct proportion to the MT length. Thus, cooperative or interfering activities between multiple motors acting on a single MT may be indicated by length-dependent changes in the motility behavior. Examining the correlation of MT length and gliding velocity (Fig. 3), we find that the slopes are not significantly different from zero, implying that there is no relationship between MT length and gliding velocity. Thus for these ensembles of dynein arms under low load, the gliding velocity is independent of the number of interacting arms, at least when the number of arms is in excess of about 49, the number of dynein arms expected to act on the shortest observed MTs $(\sim 0.5 \mu \mathrm{m})$.

\section{DISCUSSION}

The chemomechanical details of dynein motor activity are central to establishing the oscillatory bending of the flagellar axoneme. Currently there is no way to remove vertebrate dynein from the rest of the axoneme while retaining functionality for experimentation in reduced assay systems, as has been done for cytoplasmic dynein [Mallik et al., 2004] or non-mammalian, axonemal dynein [Kikushima et al., 2004]. To address this, we have established an assay in which MTs are propelled by mammalian flagellar dynein motors, which retain their attachment to extruded dMTs. In this assay, fluorescent MTs glide along axonemal dynein [Yamada et al., 1998], allowing direct observation of the activities of these nanoscopic motors. Table II summarizes several studies of dynein using a variety of assays and organisms (our current work is listed first). The maximum velocity in our gliding assays $(4.7 \pm 0.2 \mu \mathrm{m} / \mathrm{s})$ is consistent with observations of bovine sperm axoneme disintegration, where the velocity of extruding dMTs was measured in the range of about 2 to $8 \mu \mathrm{m} / \mathrm{s}$ [Bird et al., 1996]. Note that for consistency in comparisons, in the present study MTs that were observed oscillating or gliding toward the head were excluded from calculations of average velocities. We find the velocity and $K_{\mathrm{m}}$ values are close to other dynein species despite the multi-Kingdom diversity of these organisms [Kurimoto and Kamiya, 1991; Yamada et al., 1998; Shiroguchi and Toyoshima, 2001].

Our finding that the addition of ADP decreases MT gliding velocity contrasts with previous studies in which ADP increased the extent of MT sliding [Kinoshita et al., 1995], the gliding velocity [Yagi, 2000], the gliding acceleration [Kikushima et al., 2004], and the
ATPase activity [Shiroguchi and Toyoshima, 2001]. What might account for this difference? It has been hypothesized that ADP's influence is through a second nucleotide binding site on each dynein head, having a higher affinity for ADP than ATP [Shiroguchi and Toyoshima, 2001]; if this site allosterically regulates ATPase activity at the primary ATP binding site, a complex relationship could exist between dynein activity and ATP/ADP concentration. Such a relation has been hypothesized to explain the previously observed increase in ATPase activity and motility in the presence of ADP, contrary to the expected decrease in the simplest enzymatic models. As we find no evidence for increased dynein activity in the presence of ADP, it may be that mammalian dynein is simply different from protists in this respect, or it may be that ADP's influence is dominant in certain classes of dynein within an axoneme. Possibly, the effect of ADP on a subset of mammalian dyneins is overwhelmed by the unaffected motors at the density and proportions in our assays.

Unexpectedly, MTs sometimes glide in the nonphysiologic direction toward the base of the axoneme, and occasionally oscillate over very short distances. Such non-physiologic gliding presumably occurs when a MT associates with a dMT such that the plus end is near the base of the axoneme, opposite the physiologic orientation. However, additional factors must be involved since this reverse motion occurred rarely, rather than $\sim 50 \%$ expected if gliding direction was solely determined by MT landing orientation. Possibly only a subset of dynein motors can support plus-end directed motion so that "backward" motion ensues only when a MT both lands reversed from physiologic orientation and fortuitously associates with a large proportion of this subset of motors, perhaps all located on one of the dynein rows (inner or outer). This is consistent with the significant quantity of MTs that associate with a dMT but remain immobile, which then may reflect backward MTs that associate with a sufficient proportion of motors that cannot support motility in a non-physiologic direction. As the inner arms are predominantly single headed, perhaps only the inner arms are sufficiently flexible for attachment when the MT is oriented in the reverse direction. In any case, the observation of backwards movements indicates that at least a subset of motors contains sufficient compliance to associate with and propel a MT that is oriented $180^{\circ}$ from the normal direction. Though this has not been previously described for dynein, and may at first seem surprising, similar behavior has been observed for both myosin [Sellers and Kachar, 1990] and kinesin [Hunt and Howard, 1993] motors. The number of dynein motors near a MT that are capable of swiveling $180^{\circ}$ may influence whether the MT is able to glide a short distance in the wrong direction or even oscillate back 
and forth. Oscillatory behavior has also been observed for a non-mammalian axonemal dynein under loads applied using optical tweezers [Shingyoji et al., 1998]; though in that study, the MT was interacting with 1-4 dynein arms whereas in our study even the shortest oscillating MT is likely associated with $\sim 10$ times that many. We note that the oscillatory motions we observe, which typically occurred over short distances $(<1 \mu \mathrm{m}$ in either direction), could potentially be due to activities involving non-motor structures, such as elastic relaxation, or the MT interacting with a loose, wobbling dMT. Thus, these oscillations do not necessarily indicate that dynein can switch directions relative to the polarity of the translocated MT even though the MTs can oscillate along the tracks of dynein.

Since the number and arrangement of motors present on the dMT is known [Nicastro et al., 2006; Sui and Downing, 2006], we can predict the number of motors that can potentially act against the length of the gliding MT. The dependence of gliding speed on motor numbers has also been examined in studies of kinesin, myosin, and non-mammalian dynein. The speed of conventional kinesin has been found to be independent of the number of motors present [Howard et al., 1989] while the speed that myosin propels actin has been found to increase with increasing numbers of motors at low motor densities [Uyeda et al., 1991]. Likewise, the speed that nonmammalian dynein translocates MTs increases with the number of motors [Hamasaki et al., 1995]. Similar to the kinesin studies, our results indicate that for mammalian flagellar dynein the MT velocity is independent of the number of motors, at least above $\sim 49$ dynein arms. While the speed of MTs propelled by non-mammalian dynein has been shown to depend on the number of motors, this is only apparent when the estimated number of motors ranged near or less than our lowest observations. Since the drag on a sliding MT is very low, on the order of femtonewtons [Hunt et al., 1994], the independence of speed and motor number reflects an intrinsic upper limit on dynein velocity, presumably due to synchronization of hydrolysis cycles of dynein motors acting on the same MT.

Control of motion at the nanoscale is a central obstacle for developing practical nano- and micromechanical devices. A number of studies have examined the use of patterned tracks of motor proteins to guide movements [Riveline et al., 1998; Hess et al., 2001; Clemmens et al., 2003; Hoff et al., 2004; Reuther et al., 2006], but it has been a challenge keeping movements following patterned motors, especially if they are not in a straight line; the use of barriers produced through lithography is necessary to guide filament cargoes along bends [Hiratsuka et al., 2001; Moorjani et al., 2003]. The need for barriers may reflect the requirement of geometry and high density for a filament to be moved along a tight curved line. Dynein motors arrayed along dMTs are able to guide MTs along a curved track, demonstrating that with sufficient density and organization, motors can guide MTs along bends without being guided by mechanical barriers. Potentially, extruded dMTs could be used as cargo tracks for actuating nano- and micromechanical devices.

\section{CONCLUSIONS}

We have examined the movement of MTs along extruded dMTs from bovine sperm flagella. Gliding MTs displayed a range of motile behaviors, including backwards movements, that provide insight into diverse behaviors of mammalian axonemal dynein motors, and how they operate together while still attached to their natural substrate. We found that MT gliding velocity was independent of the number of dynein motors present along the dMT, and did not display evidence for increased activity due to ADP regulation. This reduced assay allows dynein motility to be studied in a controlled environment, while retaining their attachment to dMTs. This assay also allows the forces generated by mammalian dynein to be studied in ongoing biophysical assays.

\section{ACKNOWLEDGMENTS}

The authors are very grateful to: J. Damon Hoff for help editing the manuscript, answering many questions, and assay design advice; Henry T. Schek III for answering many questions and assay design advice; Kathleen Lesich for help editing the manuscript, for answering many questions, and preparing experimental samples.

\section{REFERENCES}

Avolio J, Glazzard AN, Holwill MEJ, Satir P. 1986. Structures attached to doublet microtubules of cilia-Computer modeling of thin-section and negative-stain stereo images. Proc Natl Acad Sci USA 83:4804-4808.

Bird Z, Hard R, Kanous KS, Lindemann CB. 1996. Interdoublet sliding in bovine spermatozoa: Its relationship to flagellar motility and the action of inhibitory agents. J Struct Biol 116:418-428.

Brokaw CJ. 1989. Direct measurements of sliding between outer doublet microtubules in swimming sperm flagella. Science 243: 1593-1596.

Castoldi M, Popova AV. 2003. Purification of brain tubulin through two cycles of polymerization-depolymerization in a high-molarity buffer. Protein Expr Purif 32:83-88.

Clemmens J, Hess H, Lipscomb R, Hanein Y, Bohringer KF, Matzke CM, Bachand GD, Bunker BC, Vogel V. 2003. Mechanisms of microtubule guiding on microfabricated kinesin-coated surfaces: Chemical and topographic surface patterns. Langmuir 19: 10967-10974.

Gibbons IR, Rowe AJ. 1965. Dynein-A protein with adenosine triphosphatase activity from cilia. Science 149:424-426.

Goodenough UW, Heuser JE. 1985. Substructure of inner dynein arms. Radial spokes, and the central pair projection complex of cilia and flagella. J Cell Biol 100:2008-2018. 
Hamasaki T, Holwill MEJ, Barkalow K, Satir P. 1995. Mechanochemical aspects of axonemal dynein activity studied by in vitro microtubule translocation. Biophys J 69:2569-2579.

Hess H, Clemmens J, Qin D, Howard J, Vogel V. 2001. Light-controlled molecular shuttles made from motor proteins carrying cargo on engineered surfaces. Nano Letters 1:235-239.

Hirakawa E, Higuchi H, Toyoshima YY. 2000. Processive movement of single $22 \mathrm{~S}$ dynein molecules occurs only at low ATP concentrations. Proc Natl Acad Sci USA 97:2533-2537.

Hiratsuka Y, Tada T, Oiwa K, Kanayama T, Uyeda TQP. 2001. Controlling the direction of kinesin-driven microtubule movements along microlithographic tracks. Biophys J 81:1555-1561.

Hoff JD, Cheng LJ, Meyhofer E, Guo LJ, Hunt AJ. 2004. Nanoscale protein patterning by imprint lithography. Nano Letters 4:853-857.

Howard J, Hunt AJ, Baek S. 1993. Assay of microtubule movement driven by single kinesin molecules. Methods Cell Biol 39:137-147.

Howard J, Hudspeth AJ, Vale RD. 1989. Movement of microtubules by single kinesin molecules. Nature 342:154-158.

Hunt AJ, Howard J. 1993. Kinesin swivels to permit microtubule movement in any direction. Proc Natl Acad Sci USA 90: 11653-11657.

Hunt AJ, Gittes F, Howard J. 1994. The force exerted by a single kinesin molecule against a viscous load. Biophys J 67:766-781.

Hyman A, Drechsel D, Kellogg D, Salser S, Sawin K, Steffen P, Wordeman L, Mitchison T. 1991. Preparation of modified tubulins. Methods Enzymol 196:478-485.

Kikushima K, Yagi T, Kamiya R. 2004. Slow ADP-dependent acceleration of microtubule translocation produced by an axonemal dynein. FEBS Lett 563:119-122.

Kinoshita S, Mikinoumura T, Omoto CK. 1995. Regulatory role of nucleotides in axonemal function. Cell Motil Cytoskel 32:46-54.

Kurimoto E, Kamiya R. 1991. Microtubule sliding in flagellar axonemes of Chlamydomonas-mutants missing inner-arm or outerarm dynein-Velocity-measurements on new types of mutants by an improved method. Cell Motil Cytoskel 19:275-281.

Lindemann CB, Schmitz KA. 2001. Detergent-extracted models for the study of cilia or flagella. Methods Mol Biol 161:241-252.

Lindemann CB, Fentie I, Rikmenspoel R. 1980. A selective effect of $\mathrm{Ni} 2+$ on wave initiation in bull sperm flagella. J Cell Biol $87: 420-426$.

Mallik R, Carter BC, Lex SA, King SJ, Gross SP. 2004. Cytoplasmic dynein functions as a gear in response to load. Nature 427: 649-652.

Mocz G, Gibbons IR. 2001. Model for the motor component of dynein heavy chain based on homology to the AAA family of oligomeric ATPases. Structure 9:93-103.
Moorjani SG, Jia L, Jackson TN, Hancock WO. 2003. Lithographically patterned channels spatially segregate kinesin motor activity and effectively guide microtubule movements. Nano Letters 3:633-637.

Nicastro D, Schwartz C, Pierson J, Gaudette R, Porter ME, McIntosh JR. 2006. The molecular architecture of axonemes revealed by cryoelectron tomography. Science 313:944-948.

Oiwa K, Sakakibara H. 2005. Recent progress in dynein structure and mechanism. Curr Opin Cell Biol 17:98-103.

Reuther C, Hajdo L, Tucker R, Kasprzak AA, Diez S. 2006. Biotemplated nanopatterning of planar surfaces with molecular motors. Nano Letters 6:2177-2183.

Riveline D, Ott A, Julicher F, Winkelmann DA, Cardoso O, Lacapere JJ, Magnusdottir S, Viovy JL, Gorre-Talini L, Prost J. 1998. Acting on actin: The electric motility assay. Eur Biophys J Biophys Lett 27:403-408.

Sakakibara H, Kojima H, Sakai Y, Katayama E, Oiwa K. 1999. Innerarm dynein c of Chlamydomonas flagella is a single-headed processive motor. Nature 400:586-590.

Sellers JR, Kachar B. 1990. Polarity and velocity of sliding filaments-Control of direction by actin and of speed by myosin. Science 249:406-408.

Shingyoji C, Higuchi H, Yoshimura M, Katayama E, Yanagida T. 1998. Dynein arms are oscillating force generators. Nature 393:711-714.

Shiroguchi K, Toyoshima YY. 2001. Regulation of monomeric dynein activity by ATP and ADP concentrations. Cell Motil Cytoskel 49:189-199.

Sugrue P, Avolio J, Satir P, Holwill MEJ. 1991. Computer modeling of tetrahymena axonemes at macromolecular resolution-Interpretation of electron-micrographs. J Cell Sci 98:5-16.

Sui HX, Downing KH. 2006. Molecular architecture of axonemal microtubule doublets revealed by cryo-electron tomography. Nature 442:475-478.

Uyeda TQP, Warrick HM, Kron SJ, Spudich JA. 1991. Quantized velocities at low myosin densities in an invitro motility assay. Nature 352:307-311.

Weingarten MD, Suter MM, Littman DR, Kirschner MW. 1974. Properties of depolymerization products of microtubules from mammalian brain. Biochemistry 13:5529-5537.

Yagi T. 2000. ADP-dependent microtubule translocation by flagellar inner-arm dyneins. Cell Struct Funct 25:263-267.

Yamada A, Yamaga T, Sakakibara H, Nakayama H, Oiwa K. 1998. Unidirectional movement of fluorescent microtubules on rows of dynein arms of disintegrated axonemes. J Cell Sci 111:93-98. 